\title{
Kiwi Seed Test for Detection of Enterocutaneous Fistula
}

\author{
Michael Knoop • Georg Fritzsch
}

Published online: 18 December 2014

(C) Société Internationale de Chirurgie 2014

\section{To the Editor}

We read with interest the paper of Gui et al. [1] on vacuumassisted wound care for closure of low- and high-output enterocutaneous fistulas. We would like to share our observation of a simple bed-side test for the detection of low-output enterocutaneous fistulas.

Up to $5 \%$ of patients undergoing GI-tract surgery develop an anastomotic leak with the need of multiple reoperations, prolonged hospital stay, and a fatal outcome up to $24 \%$ in this heterogenous subgroup [2]. Nearly $80 \%$ of all leakages and fistulas can be visualized and verified by examination of drainage fluid, ultrasound, endoscopy, and instillation of water-soluble contrast media or methylene blue [3]. While high-output fistulas require surgical intervention, low-output fistulas, however, with a drainage volume of less than $200 \mathrm{ml} /$ day often close spontaneously, and oral feeding is well tolerated [4]. High-output fistulas are easy to diagnose while low-output fistulas are difficult to prove.

Three patients with low-output fistulas in the abdominal incision (62-years-old male with fistula in the right subcostal incision after open cholecystectomy and acute pancreatitis; 32-years-old male with fistula of the small bowel after multiple relaparotomies; 78-years-old female after sigmoid resection for diverticulitis) were treated conservatively in the last 18 months. These patients had oral nutrition without increase of fistula output. In all cases,

M. Knoop $(\bowtie) \cdot$ G. Fritzsch

Department of General and Visceral Surgery, Klinikum Frankfurt (Oder), University of Berlin (Charite) Teaching Hospital, Muellroser Chaussee 7, 15236 Frankfurt (Oder), Germany

e-mail: mdrknoop@aol.com however, it was impossible to document a real fistula by clinical and radiological examination (fistulography, contrast medium enema). After intake of kiwi fruit, the seeds appeared in the fistula fluid documenting a duodenal, a small bowel, and a small anastomotic leak of the colon, respectively. All fistulas closed spontaneously.

This observation shows that the kiwi test represents a simple, accurate, convenient, and inexpensive tool to diagnose low-output enterocutaneous fistulas after abdominal surgery. A similar observation has been made for the detection of enterovesical fistulas with the use of poppy seed [5].

\section{References}

1. Gui D, Pepe G, Callari C et al (2014) Vacuum-assisted wound care (V.A.C.) for enteric fistula closure: how we do it. World J Surg. doi:10.1007/s00268-014-2739-0

2. Pickleman J, Watson W, Cunningham J, Fisher SG, Gamelli R (1999) The failed gastrointestinal anastomosis: an inevitable catastrophe? J Am Coll Surg 188:473-482

3. Zirngibl H, Schmidt J (1997) Concepts for recognition and securing anastomotic wound dehiscence of the gastrointestinal tract. Zentralbl Chir 122:20-24

4. Rahbour G, Gabe SM, Ullah MR, Thomas GP, Al-Hassi HO, Yassin NA et al (2013) Seven-year experience of enterocutaneous fistula with univariate and multivariate analysis of factors associated with healing: development of a validated scoring system. Colorectal Dis 15:1162-1170

5. Kwon EO, Armenakas NA, Scharf SC, Panagopoulos G, Fracchia JA (2008) The poppy seed test for colovesical fistula: big bang, little bucks! J Urol 179:1425-1427 